\title{
Estudio Comparativo del Poder Lubricante y Estabilidad Oxidativa entre el Aceite de Ajonjolí y Aceite Mineral $\mathbf{3 6 0}$
}

\author{
Arnoldo E. Delgado(1,2)* y William A. Aperador ${ }^{(1)}$ \\ (1) Universidad Militar Nueva Granada, Facultad de Ingeniería, Departamento de Ingeniería Mecatrónica, \\ Carrera 11 No. 101-80. Bogotá-Colombia. (e-mail: william.aperador@unimilitar.edu.co) \\ (2) Escuela Colombiana de Ingeniería Julio Garavito, Facultad de Ingeniería, Departamento de Ingeniería \\ Industrial, Carrera 45 No. 205-59. Bogotá-Colombia. (e-mail: articulopublish@gmail.com;
}

${ }^{*}$ Autor a quien debe ser dirigida la correspondencia

Recibido Nov. 21, 2013; Aceptado Ene. 15, 2014; Versión final recibida Mar. 17, 2014

\begin{abstract}
Resumen
Se presenta un estudio comparativo del poder lubricante y la estabilidad oxidativa entre el aceite de ajonjolí y un aceite mineral, ambos sin aditivos. Se verificó el poder lubricante superior del aceite vegetal sobre el aceite mineral mediante ensayo de desgaste preventivo y presión extrema en un tribómetro cuatro bolas. Según el ensayo de absorción atómica, hay presencia de trazas metálicas provenientes de las esferas que están en contacto con el aceite de ajonjolí durante el desarrollo de la prueba de desgaste preventivo. Los metales catalizan la oxidación del aceite durante el ensayo de calorimetría diferencial de barrido disminuyendo la temperatura de inicio de oxidación. El ensayo de desgaste preventivo degradó parcialmente el aceite de ajonjolí aumentó el valor del peróxido. Este cambio en el aceite no fue posible detectarlo mediante espectroscopia infrarroja con transformada de Fourier y acidez. La estabilidad del aceite mineral es superior a la del aceite vegetal.
\end{abstract}

\section{Comparative Study of the Lubricating Power and Oxidative Stability between Sesame Oil and Mineral Oil 360}

\begin{abstract}
A comparative study of the lubricant properties and oxidative stability of sesame oil and a mineral oil, without additives is presented. The superior power of vegetable oil lubricant compared to that of mineral oil was verified by preventive wear tests and extreme pressure in a four-ball tribometer. According to atomic absorption test, there are traces of metals in the areas that are in contact with the sesame oil during the development of the preventative wear test. These particles catalyze the oxidation of the oil during the differential scanning calorimetry test, decreasing the oxidation starting temperature. The preventive wear test partially degraded the sesame oil and increased the peroxide value. This change in the oil was not possible to find by Fourier transform with infrared spectroscopy and acidity. The stability of the mineral oil is superior to that of the vegetable oil.
\end{abstract}

Keywords: sesame, tribology, oxidative stability, four-ball tester, DSC. 


\section{INTRODUCCIÓN}

La mayoría de los lubricantes provienen de origen petroquímico y sintético, son tóxicos y no biodegradables, generan contaminación durante su fabricación y uso. El creciente deterioro ambiental y el incremento del precio del petróleo, como resultado del agotamiento de las reservas de este recurso no renovable, han incentivado el desarrollo de "lubricantes verdes" (Lafont et al., 2011; Kabuya et al., 1995; Menezes et al., 2012). Los aceites vegetales son renovables, biodegradables, no tóxicos, poseen la mayoría de las propiedades requeridas para lubricación, tales como alto índice de viscosidad, baja volatilidad, buena lubricidad y son excelentes disolventes para aditivos y contaminantes polares. Sin embargo, como desventajas poseen baja estabilidad térmica, oxidativa e hidrolítica, y escasa fluidez a bajas temperaturas (Sathwik et al., 2012; Jayadas et al., 2006).

Las propiedades de los aceites vegetales están determinadas por su composición de ácidos grasos. Un alto contenido de ácidos grasos insaturados disminuye la estabilidad térmica-oxidativa, mientras que una mayor proporción de ácidos grasos saturados de cadena larga conduce a una disminución de la fluidez en frío (Jayadas et al., 2006). Los aceites vegetales tienen una estabilidad oxidativa pobre, debido principalmente a la presencia de protones bis-alílicos. Por acción de la luz, la temperatura y el oxígeno, estos sitios activos son muy susceptibles al ataque de radicales, y forman compuestos oxigenados polares que resultan en depósitos insolubles y en el aumento de la acidez, la corrosión, la viscosidad y la volatilidad del aceite (Bart et al., 2013; Sharma et al., 2007). Sin embargo, el mejoramiento de las propiedades térmicas y lubricantes de los aceites vegetales se puede lograr mediante modificaciones químicas tales como epoxidación, transesterificación o hidrogenación selectiva (Biresaw et al., 2011; Alves et al., 2013) y el uso de aditivos de tipo antioxidante, detergente, dispersante, de extrema presión o anti-desgaste (Guala et al., 2009; Sharma et al., 2007; Minami et al., 1998).

El aceite de ajonjolí tiene ventajas en la formulación de lubricantes verdes con respecto a otros aceites vegetales; por ejemplo, tiene mejor fluidez a temperaturas bajas debido a la alta concentración de dobles enlaces olefínicos (Jayadas et al., 2006); los ácidos grasos insaturados predominantes son ácido oleico y ácido linoleico (ver tabla 1) (Sathwik et al., 2012; Mannekote et al., 2012). Además, el aceite de ajonjolí contiene una proporción significativa de antioxidantes naturales, conocidos como lignanos (tales como sesamina, sesamolina y sesamol), que lo protegen parcialmente frente a la oxidación (Sathwik et al., 2012; $\mathrm{Wu}, 2007)$.

Debido a que la oxidación de los aceites vegetales es una reacción exotérmica, los cambios en la entalpía de este proceso químico se pueden medir por Calorimetría Diferencial de Barrido (DSC), durante el calentamiento de la muestra en una atmósfera oxidante. La temperatura de comienzo de la oxidación (TONSET) y el tiempo de inducción a la oxidación (To), obtenidos por extrapolación a partir de curvas DSC isotérmicas de oxidación, pueden relacionarse con la estabilidad oxidativa de los aceites (Tan et al., 2002; Pardauil et al., 2011). En comparación con los métodos convencionales, como la prueba Rancimat para hallar los índices de estabilidad oxidativa (Honary et al., 2011), DSC es una técnica reproducible que consume menos tiempo de análisis y menos cantidad de muestra. Por lo tanto, el estado o la calidad de un aceite vegetal usado en lubricación, se puede evaluar determinando su estabilidad oxidativa por DSC.

En esta investigación, se comparó tanto el poder lubricante como la estabilidad oxidativa del aceite refinado de ajonjolí con un aceite mineral, ambos sin aditivos. Las propiedades tribológicas se realizaron según el método cuatro bolas para las pruebas de Desgaste Preventivo (DP) y Presión Extrema (PE). La estabilidad oxidativa de los aceites se determinó mediante espectroscopia FT-IR, valor peróxido, acidez, DSC y espectroscopia de absorción atómica.

Tabla 1: Composición de ácidos grasos del aceite de ajonjolí

\begin{tabular}{|c|c|c|c|}
\hline Nombre del ácido graso & Fracción (\%) & Nombre del ácido graso & Fracción (\%) \\
\hline Láurico & 0,4 & Oleico & 41,4 \\
\hline Mirístico & 0,2 & Linoleico & 40,4 \\
\hline Palmítico & 10,7 & Linolénico & 0,4 \\
\hline Palmitoleico & 0,2 & Araquídico & 0,4 \\
\hline Esteárico & 5,2 & Behénico & 0,6 \\
\hline
\end{tabular}




\section{MATERIALES Y MÉTODOS}

\section{Materiales}

Aceite de ajonjolí refinado, densidad: $0,98 \mathrm{~g} / \mathrm{cm}^{3}$, índice de acidez: $0,33 \mathrm{mg}-\mathrm{KOH} / \mathrm{g}$, viscosidad cinemática: $34,64 \mathrm{cSt}$. Aceite mineral 360 , densidad: $0,86 \mathrm{~g} / \mathrm{cm}^{3}$, viscosidad cinemática: $52,36 \mathrm{cSt}$. Para los ensayos de tribología se emplearon esferas de acero de aleación de cromo AISI 52100, con 12,7 mm de diámetro, dureza de 60-66 HRC. La composición porcentual química de las esferas según certificado de calidad es: carbono: 0,96; manganeso: 0,33; silicio: 0,26; azufre: 0,004; fósforo: 0,011; cromo: 1,48; níquel: 0,05; cobre: 0,110 y titanio 0,0016 .

\section{Ensayos tribológicos}

La prueba de desgaste preventivo se realizó de acuerdo a la norma ASTM D 4172-94, con las siguientes condiciones: $75^{\circ} \mathrm{C}, 1200 \mathrm{rpm}$, carga de 147 Newton y tiempo de 60 minutos. Los parámetros para evaluar la lubricidad de los aceites fueron el diámetro promedio de la huella de desgaste (DPH) y el coeficiente de fricción (f). El DPH se midió en milímetros usando un microscopio óptico. La determinación de las propiedades de presión extrema: Carga Anterior al Desgaste Visible y Punto de Soldadura se determinaron según la norma ASTM D 2783-03, con las siguientes condiciones: temperatura: $23{ }^{\circ} \mathrm{C}, 1760 \mathrm{rpm}$, cargas variables: $59,78,98,127,157,196,235,314 \mathrm{~N}$, tiempo de cada prueba de 10 segundos. Todos los ensayos se realizaron en un tribómetro cuatro bolas (ver fig. 1).

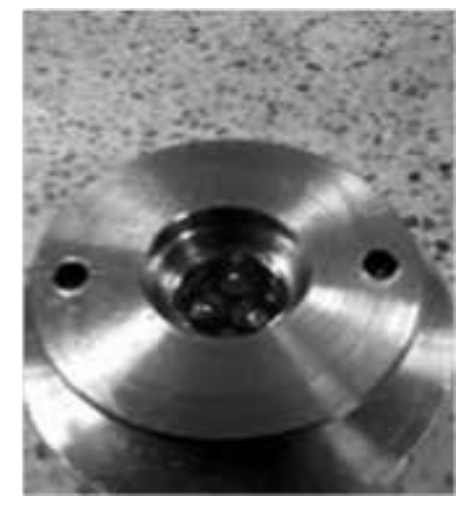

Fig. 1: Tribómetro cuatro bolas

\section{Espectroscopia de absorción atómica}

Mediante esta prueba se determinó la presencia de partículas metálicas suspendidas en el aceite, las cuales provienen del ensayo de desgaste preventivo. La técnica se llevó a cabo por absorción en llama, nebulizando la muestra y luego diseminándola en forma de aerosol dentro de una llama de aire acetileno. Este análisis fue realizado por el laboratorio Cromanal® Ltda.

\section{Ensayos para determinar la estabilidad oxidativa del aceite}

\section{Espectroscopia FT-IR}

Se realizó con un espectrómetro Nicolet iS10 (Thermo Fisher Scientific) equipado con detector de sulfato de triglicina deuterada (DTGS) y beamsplitter de $\mathrm{KBr}$. Con la técnica de muestreo de reflectancia total atenuada horizontal (HATR), la muestra se colocó en contacto directo con el cristal de seleniuro de zinc (ZnSe) del accesorio Smart ARK ${ }^{\mathrm{TM}}$ de múltiple rebote a temperatura de $23^{\circ} \mathrm{C}$. Los espectros FT-IR fueron adquiridos con una resolución de $4 \mathrm{~cm}^{-1}$, número de scans de 32 y rango espectral de $4000-650 \mathrm{~cm}^{-1}$. El análisis de los espectros se realizó con el software OMNIC 9.1.27 (Thermo Fisher Scientific Inc.).

\section{Calorimetría Diferencial de Barrido - DSC}

Se llevó a cabo en el equipo DSC Serie Q20 de TA Instruments ${ }^{\circledR}$. El instrumento se calibró con indio puro (punto de fusión $156,60^{\circ} \mathrm{C} ; \Delta \mathrm{H}_{\mathrm{f}}=28,45 \mathrm{~J} / \mathrm{g}$ ), y la línea base se obtuvo con un crisol de aluminio vacío. Las muestras de aceite de 10-12 mg se pesaron en crisoles de aluminio abiertos y se colocaron en la celda DSC Tzero ${ }^{\circledR}$ con un crisol vacío como referencia. Las muestras de aceite se purgaron con un flujo de 50 $\mathrm{mL} / \mathrm{min}$ de aire, y se llevaron desde $10^{\circ} \mathrm{C}$ hasta a $250^{\circ} \mathrm{C}$ con una velocidad de calentamiento de $10^{\circ} \mathrm{C} / \mathrm{min}$. A partir de las curvas de calentamiento flujo de calor $(\mathrm{W} / \mathrm{g})$ en función de la temperatura, se determinaron las 
temperaturas de comienzo de la oxidación (TONSET). Los datos se analizaron con el software Universal Analysis 2000, versión 4.5A (TA Instruments $\left.{ }^{\circledR}\right)$.

Valor peróxido (VP) y acidez

El VP de las muestras de aceite se determinó de acuerdo con el método establecido por la Asociación de Comunidades Analíticas - AOAC 965.33. Por lo tanto, el VP se midió como la cantidad de sustancias en la muestra que oxidan el yoduro de potasio en las condiciones de prueba, expresado en mili-equivalentes de peróxido por kilogramo (mEq peróxido/kg). La acidez se evaluó según norma ISO 660:2009 empleando el método del solvente frío usando indicador, debido a que es la metodología más adecuada para aceites que no tienen un color muy fuerte, se expresa en porcentaje de ácido oleico.

\section{RESULTADOS Y DISCUSIÓN}

\section{Ensayos de Desgaste Preventivo (DP)}

Los valores correspondientes al diámetro promedio de la huella de desgaste y el coeficiente de fricción son menores para el aceite de ajonjolí con respecto al aceite mineral 360 (ver fig. 2 y tabla 2). La fig. 3 muestra las imágenes de las huellas de desgaste, observadas en un microscopio óptico, al finalizar el ensayo DP se observa mayor desgaste sobre la superficie metálica de las esferas lubricadas con el aceite mineral 360.

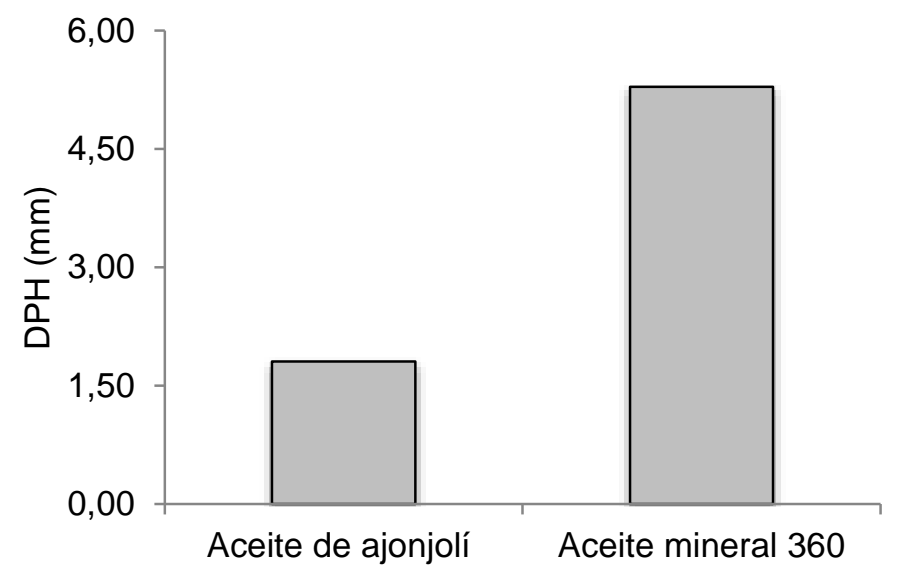

Fig. 2: Diámetros promedio de la huella de desgaste obtenidos en los ensayos de desgaste preventivo

Tabla 2: Coeficientes de fricción, ensayo de desgaste preventivo

\begin{tabular}{|c|c|}
\hline \multicolumn{1}{|c|}{ Aceite } & Coeficiente de fricción \\
\hline Aceite de ajonjolí & 7,1 \\
\hline Aceite mineral 360 & 7,7 \\
\hline
\end{tabular}

El aceite de ajonjolí consiste en una mezcla de triglicéridos formados por ácidos grasos mientras que el aceite mineral está constituido por mezclas de hidrocarburos alifáticos, motivo por la cual el aceite de ajonjolí presenta una mayor capacidad de lubricación. La naturaleza polar del aceite de ajonjolí le da mayor afinidad por las superficies metálicas que el aceite mineral no polar (Sathwik et al., 2012). Los triglicéridos son moléculas anfifílicas con cabeza polar hidrofílica y cadenas hidrocarbonadas no polares e hidrofóbicas. El extremo polar está formado por grupos éster. Este grupo funcional hace que el aceite de ajonjolí presente mejor lubricidad que el aceite mineral. Las cabezas polares de las cadenas de ácidos grasos se unen a las superficies metálicas mediante un proceso de adsorción que permite la formación de una película de mono capa con el extremo no polar de los ácidos grasos sobresaliendo lejos de la superficie del metal. Las cadenas hidrocarbonadas de los ácidos grasos ofrecen una superficie de deslizamiento que evita el contacto directo de metal a metal (Bart et al., 2013). De esta manera, la presencia de las moléculas adsorbidas reduce el coeficiente de fricción. 

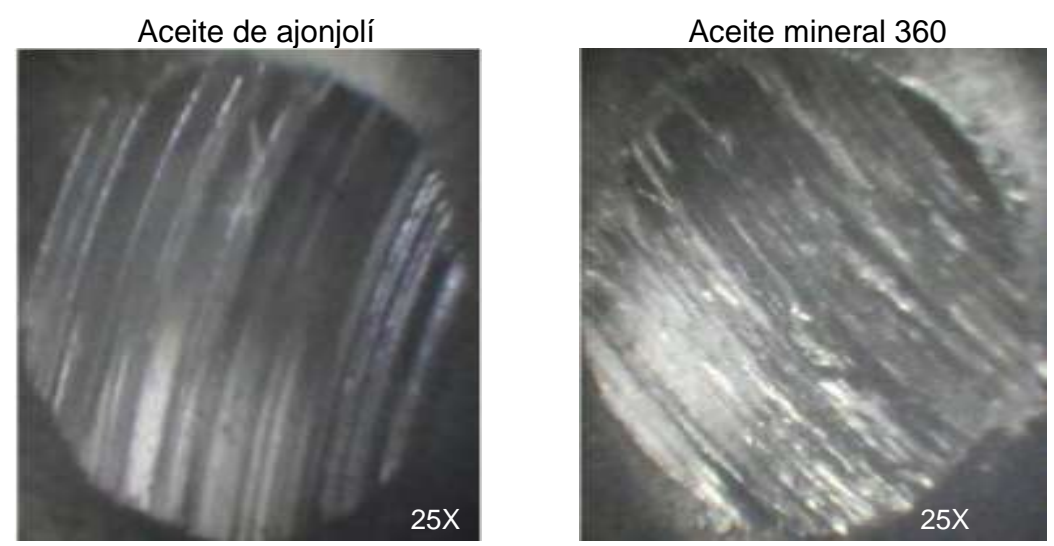

Fig. 3: Diámetro de huellas. Ensayo de Desgaste Preventivo - DP

\section{Propiedades de Presión Extrema (PE)}

El desempeño de los aceites en condiciones de extrema presión, se evaluó en términos de la Carga Anterior al Desgaste Visible y el Punto de Soldadura. En la fig. 4 y 5 se muestra el comportamiento del diámetro promedio de la huella de desgaste en función de la carga aplicada, se observa un cambio de pendiente a partir de la carga anterior al desgaste visible. Esta carga se encuentra en $127 \mathrm{~N}$ para el aceite de ajonjolí y en $98 \mathrm{~N}$ para el aceite 360 .

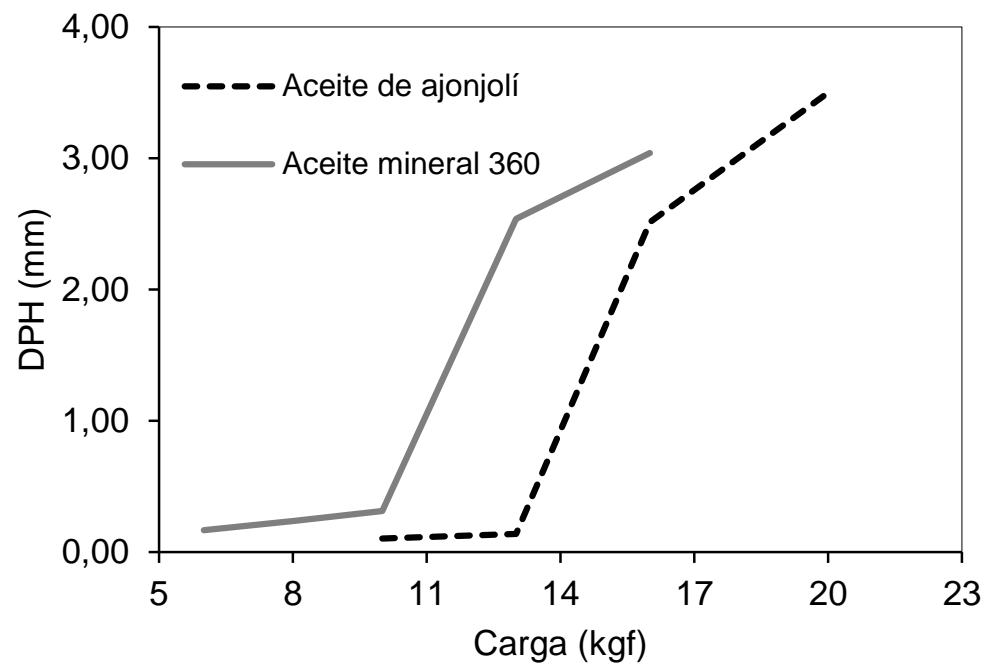

Fig. 4: Diámetro promedio de la huella de desgaste en función de la carga aplicada, ensayo de extrema presión

El aceite de ajonjolí presenta un punto de soldadura de $235 \mathrm{~N}$, mientras que el aceite mineral tiene el punto de soldadura de $196 \mathrm{~N}$ (fig. 5). El aceite de ajonjolí tiene mejor desempeño en condiciones de extrema presión que el aceite mineral 360; es decir, posee mayor poder lubricante. El aceite vegetal está constituido por moléculas de alta polaridad que le permiten interacciones más fuertes con las superficies lubricadas y por tanto mayor capacidad de adsorción que inhibe el contacto de metal a metal (Kabuya et al., 1995; Bart et al., 2013). En la fig. 6, se observa la formación de huella, en la parte superior de cada una de las imágenes está el valor de la fuerza aplicada justo antes del desgaste visible.

Las imágenes del punto de soldadura (ver fig. 7) muestran evidente deformación de la esfera, debido a la presión ejercida, lo cual produce la unión de las esferas, en la parte superior de cada figura está el valor de la fuerza a la que se produce el punto de soldadura. 


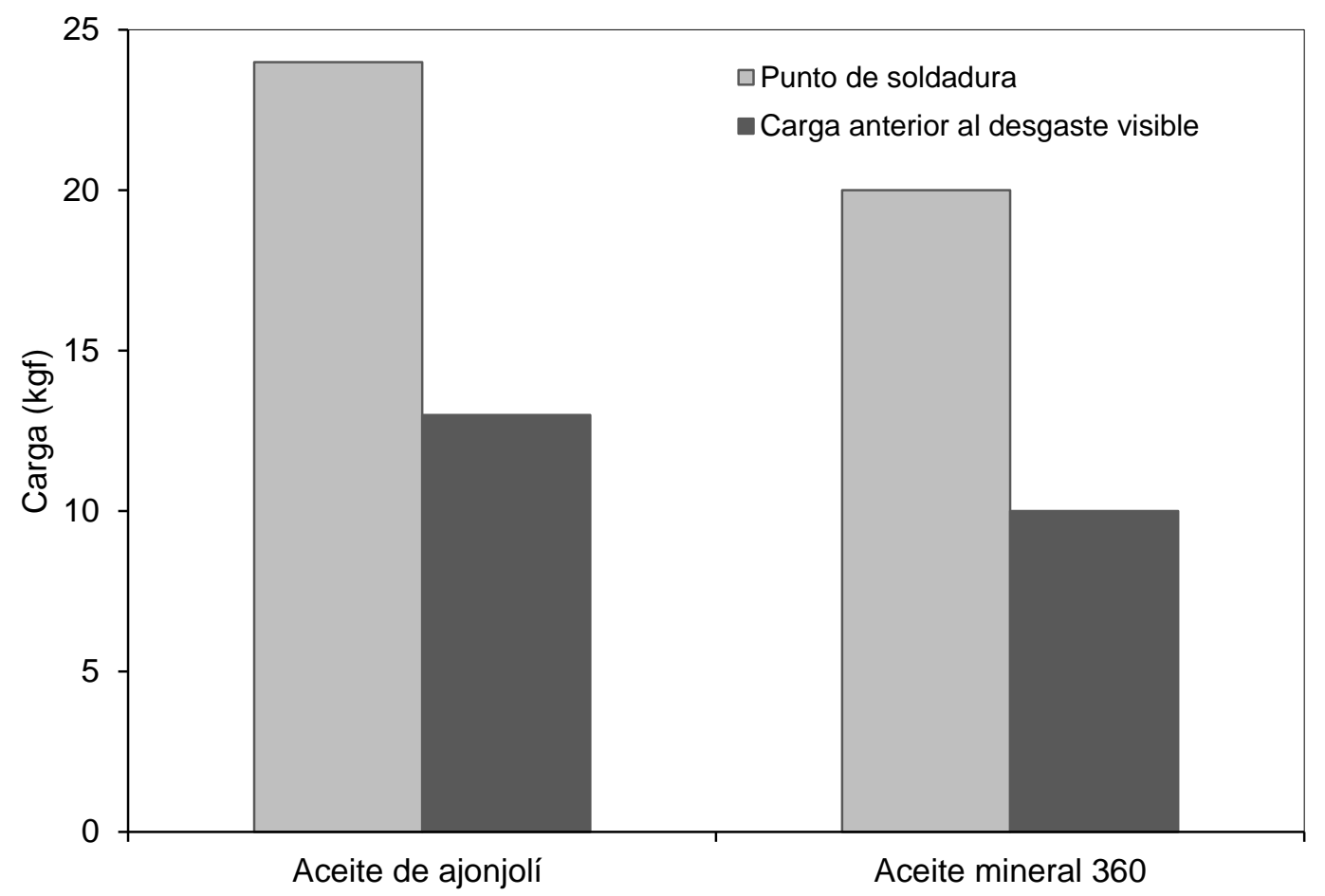

Fig. 5: Propiedades de Presión Extrema

127 Newton

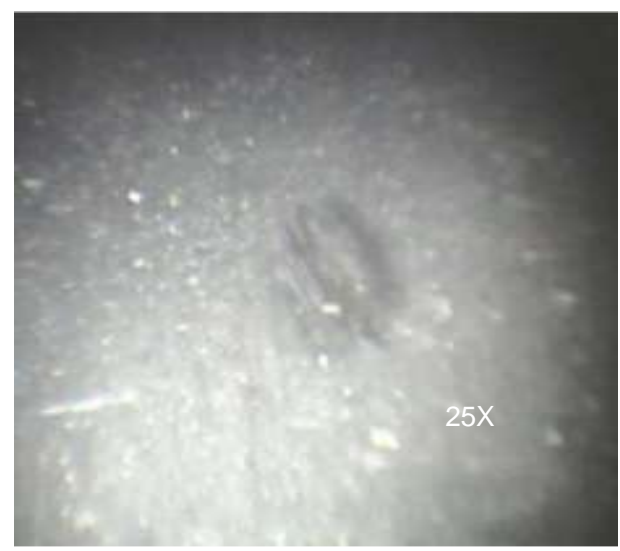

Ajonjoli
98 Newton

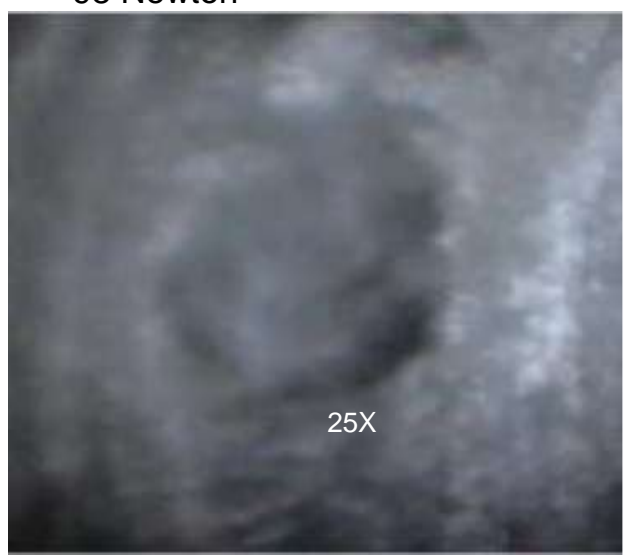

Aceite mineral 360

Fig. 6: Imagen de diámetro de huella. Carga anterior al desgaste visible

$236 \mathrm{~N}$

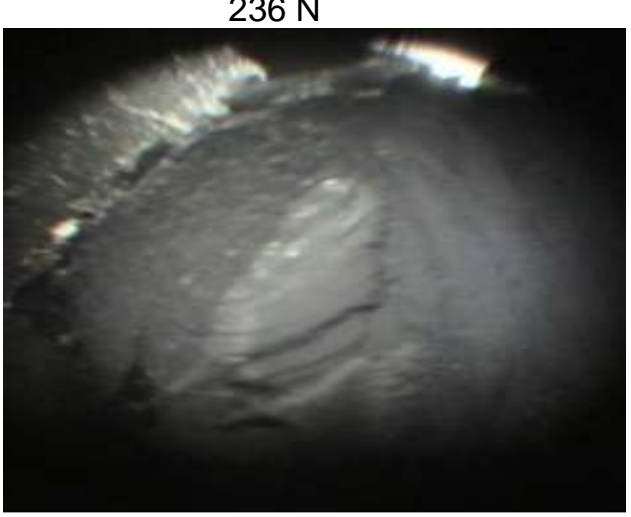

Ajonjolí

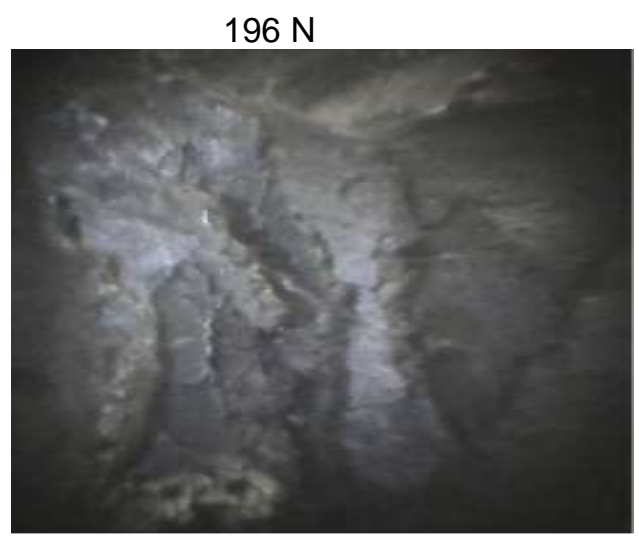

Aceite mineral 360

Fig. 7: Imagen del punto de soldadura 


\section{Espectroscopia de absorción atómica}

La muestra de aceite de ajonjolí proveniente de la prueba DP se observó turbia con respecto a la sometida a ensayo de PE. Por tal motivo al aceite de ajonjolí con DP se le realizó prueba de espectroscopia de absorción atómica para determinar la presencia de trazas metálicas. El ensayo se le realizó para los metales de cromo y manganeso. El reporte suministrado por el laboratorio Cromanal® Ltda. indica que la muestra analizada no logró ser cuantificable debido a la baja concentración de los metales, sin embargo cualitativamente presenta absorción a la longitud de onda de $357,9 \mathrm{~nm}$ y $279,5 \mathrm{~nm}$ características de cromo y manganeso respectivamente.

\section{Estabilidad oxidativa de los aceites usados en el ensayo DP}

El cambio organoléptico del aceite vegetal sometido a DP estuvo relacionado con el de la degradación y también fue debido al color impartido por las trazas de partículas metálicas que se desprendieron de las esferas y quedaron suspendidas en el aceite. A continuación se analiza la estabilidad oxidativa de los aceites de ajonjolí y mineral 360 usados en el ensayo DP, es importante recordar que en esta prueba, los aceites fueron sometidos a fricción con carga de $147 \mathrm{~N}$ a $75^{\circ} \mathrm{C}$ durante una hora.

\section{Espectroscopia FT-IR}

En el espectro IR del aceite de ajonjolí extrafino se observan las siguientes bandas: en $3008 \mathrm{~cm}^{-1}$ : vibración del estiramiento $=\mathrm{C}-\mathrm{H}$ (cis) de dobles enlaces olefínicos. En 2923 y $2853 \mathrm{~cm}^{-1}$ : vibraciones asimétricas y simétricas del estiramiento $-\mathrm{C}-\mathrm{H}$ del grupo metileno $\left(\mathrm{CH}_{2}\right)$, respectivamente. En $1743 \mathrm{~cm}^{-1}$ : estiramiento $\mathrm{C}=\mathrm{O}$ característico del grupo éster de los triglicéridos. En $1464 \mathrm{~cm}^{-1}$ : vibraciones atribuidas a la flexión -C$\mathrm{H}$ de los grupos alifáticos $\mathrm{CH}_{2}$ y $\mathrm{CH}_{3}$. En $1377 \mathrm{~cm}^{-1}$ : vibración simétrica de la flexión -C-H del grupo metilo $\left(\mathrm{CH}_{3}\right)$. Las bandas en 1238, 1159 y $1097 \mathrm{~cm}^{-1}$ se pueden asignar a las vibraciones del estiramiento del grupo -C-O en los ésteres. Finalmente, la banda en $722 \mathrm{~cm}^{-1}$ es la superposición de la vibración de balanceo (rocking) de $\mathrm{CH}_{2}$ y la vibración de la flexión fuera del plano de las olefinas cis-disustituidas (Guillén et al., 1997). Estos resultados demuestran que el aceite de ajonjolí consiste en una mezcla de triglicéridos formados por ácidos grasos insaturados y saturados (ver tabla 1). En la fig. 8 se muestran los espectros FTIR del aceite de ajonjolí antes y después del ensayo DP, no se detectan nuevas absorciones originadas por productos de oxidación ni desplazamientos en los valores de frecuencia de las bandas. Es evidente que mediante espectroscopia FT-IR no fue posible determinar si el aceite de ajonjolí usado en las pruebas de DP presentó degradación.

En el espectro IR del aceite mineral 360 se observan únicamente bandas correspondientes a hidrocarburos alifáticos (fig. 9), en $2953 \mathrm{~cm}^{-1}$ aparece la banda de vibración asimétrica del estiramiento -C-H del grupo metilo $\left(\mathrm{CH}_{3}\right)$, las bandas en 2921 y $2852 \mathrm{~cm}^{-1}$ corresponden a las vibraciones asimétricas y simétricas del estiramiento $-\mathrm{C}-\mathrm{H}$ del grupo metileno $\left(\mathrm{CH}_{2}\right)$ respectivamente, las vibraciones en $1459 \mathrm{~cm}^{-1}$ se atribuyen a la flexión -C-H de los grupos $\mathrm{CH}_{2}$ y $\mathrm{CH}_{3}$. En $1377 \mathrm{~cm}^{-1}$ : vibración simétrica de la flexión $-\mathrm{C}-\mathrm{H}$ del grupo $\mathrm{CH}_{3}$. Finalmente, la banda en $722 \mathrm{~cm}^{-1}$ es la superposición de la vibración del balanceo (rocking) de $\mathrm{CH}_{2}$. Estos resultados demuestran que el aceite mineral consiste en una mezcla de hidrocarburos alifáticos. En la fig. 9 se muestran los espectros FT-IR del aceite mineral 360 antes y después del ensayo DP.

\section{$D S C$}

Es evidente la disminución de la estabilidad oxidativa del aceite de ajonjolí usado en el ensayo de DP, la temperatura de inicio de oxidación disminuye s en $70,251^{\circ} \mathrm{C}$ con respecto al aceite virgen, CUya TONSET es $189,01^{\circ} \mathrm{C}$ (TONSET del aceite usado es $118,759^{\circ} \mathrm{C}$ ), (fig. 10), la disminución de la estabilidad oxidativa fue causada por la presencia de partículas metálicas, y por la degradación inicial de aceite durante la prueba DP. El aceite vegetal contiene protones bis-alílicos susceptibles al ataque de radicales que posteriormente forman compuestos oxigenados polares que resultan en depósitos insolubles y en la disminución de la estabilidad oxidativa, adicionalmente los metales catalizan la oxidación de las cadenas de ácidos grasos; es decir, aceleran la degradación del aceite vegetal (Nunes et al., 2011). Como ensayo confirmativo se realizó DSC a una muestra centrifugada del aceite de ajonjolí usado en DP, el valor obtenido de TONSET fue $156,99^{\circ} \mathrm{C}$ el cual es un valor intermedio entre los dos resultados previamente mencionados, lo que confirma el efecto negativo de los metales sobre la estabilidad oxidativa de aceites vegetales. 


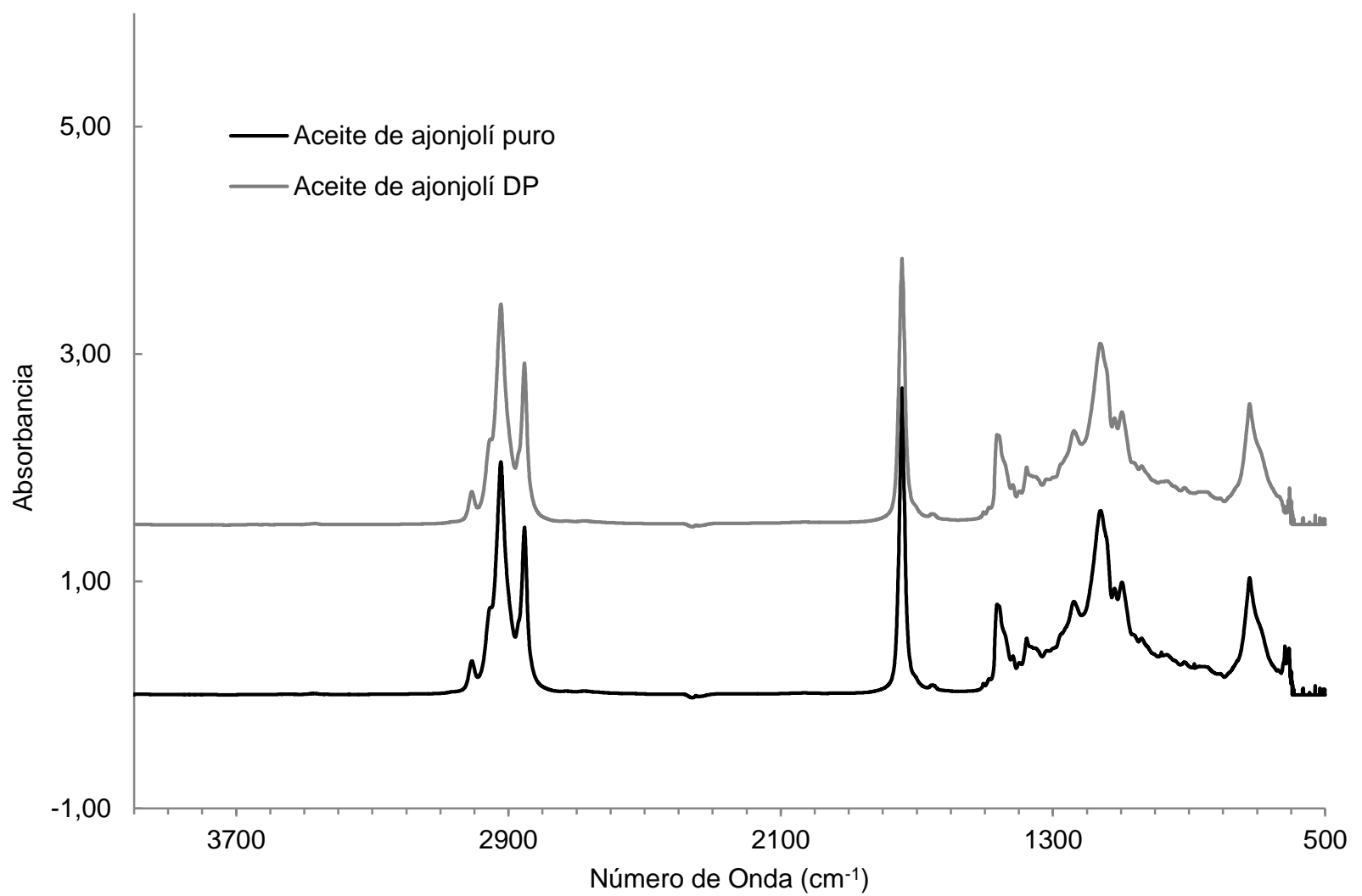

Fig. 8: Espectros FT-IR del aceite de ajonjolí virgen y usado en la prueba DP

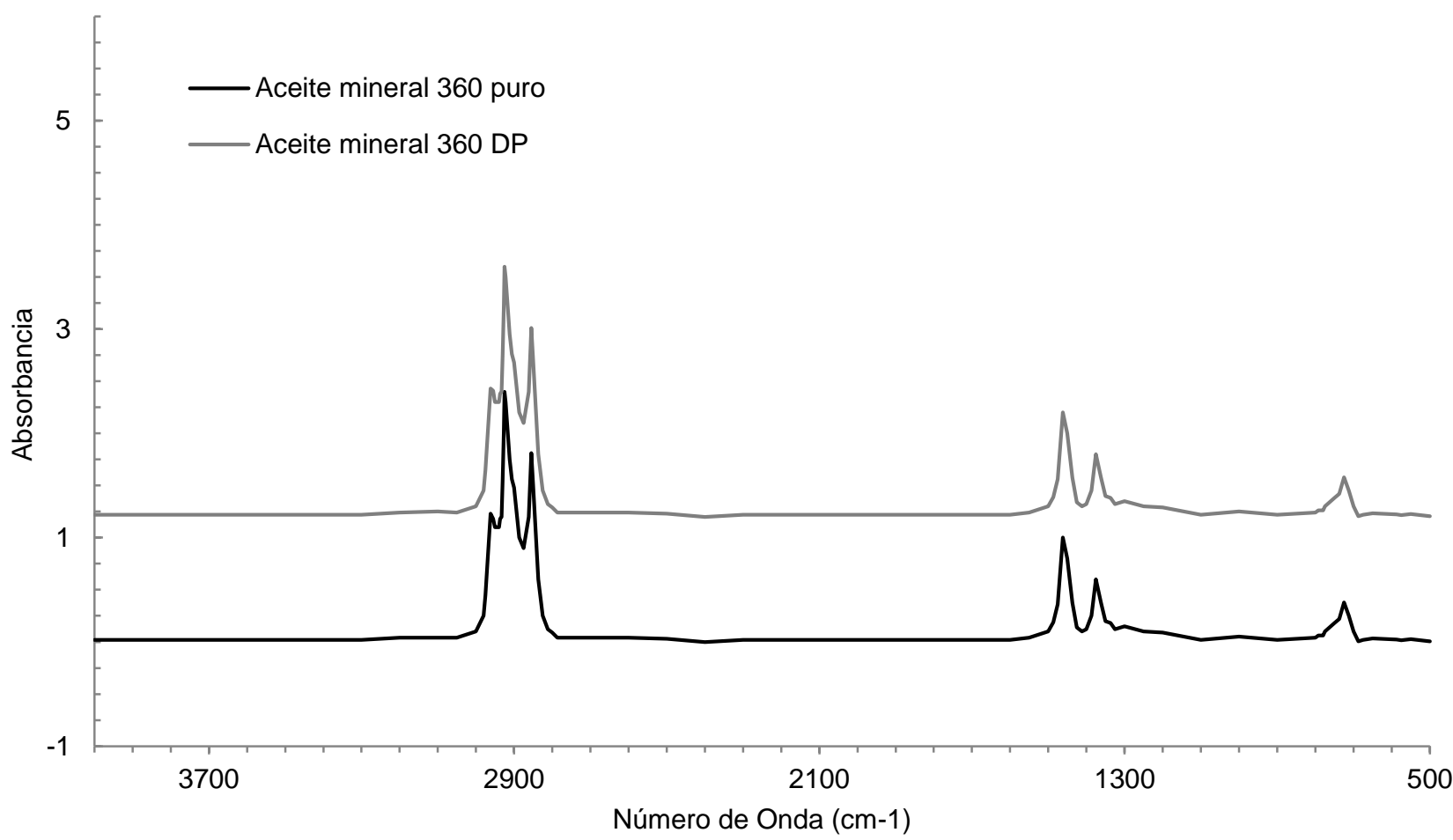

Fig. 9: Espectros FT-IR del aceite mineral 360 virgen y usado en la prueba DP

El aceite mineral es más estable a la oxidación puesto que sus cadenas hidrocarbonadas saturadas no tienen sitios fácilmente oxidables. Como se observa en la fig. 11, en el aceite 360 no hay disminución significativa de la TONSET, luego del ensayo de DP presenta una TONSET de $216,99^{\circ} \mathrm{C}, 9,55^{\circ} \mathrm{C}$ por debajo de la TONSET del aceite virgen $\left(226,54^{\circ} \mathrm{C}\right)$. 


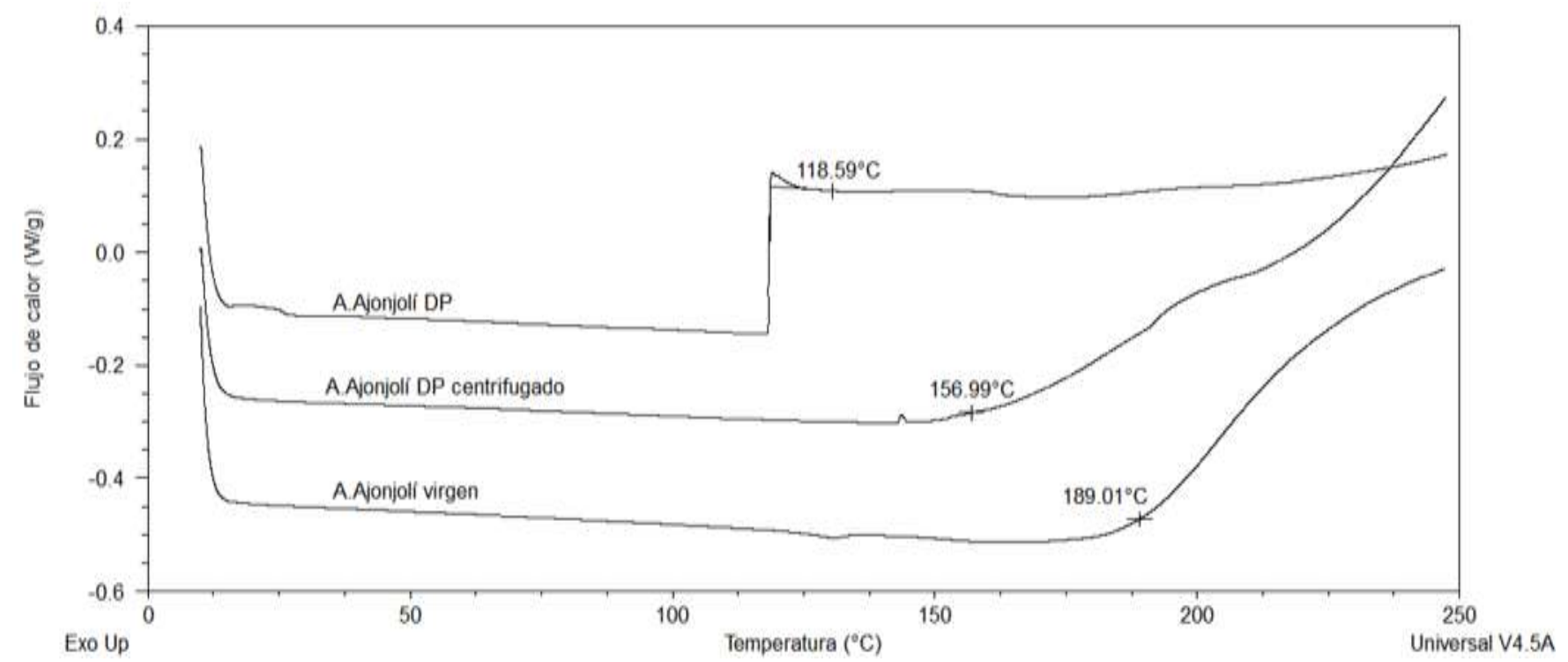

Fig. 10: Curvas DSC de oxidación en aire del aceite de ajonjolí virgen y usado, después de los ensayos de desgaste preventivo (DP) y centrifugación

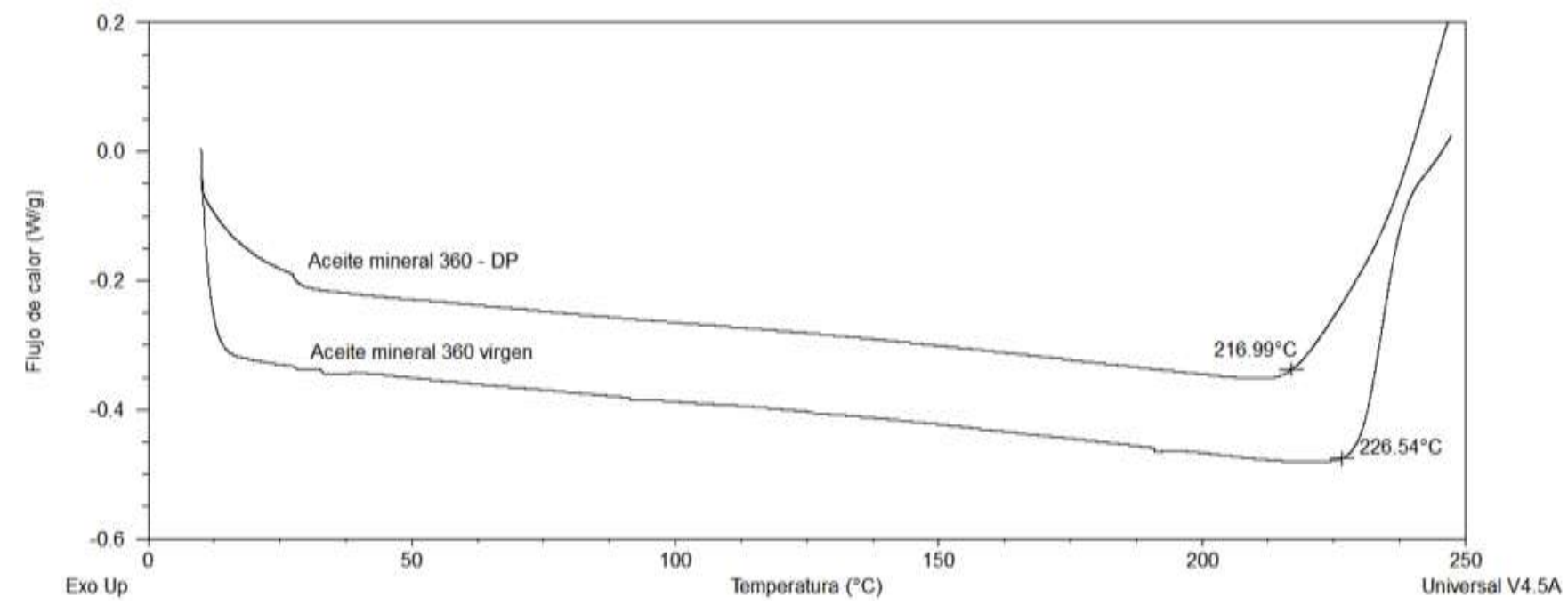

Fig. 11: Curvas DSC de oxidación en aire del aceite mineral 360 virgen y usado, después del ensayo de desgaste preventivo (DP)

Valor Peróxido (VP) y Acidez del aceite de ajonjolí DP

La reacción de auto-oxidación en aceites vegetales es imposible de evitar sin la presencia de antioxidantes y se inicia por iones metálicos o por energía térmica o luminosa en presencia de oxígeno. Existen métodos para determinar el estado oxidativo de aceites, siendo los más utilizados valor peróxido y la acidez (Barrera, 1998). En esta investigación la acidez no permitió cuantificar la oxidación del aceite vegetal debido a que se mantuvo constante antes y después de la prueba de DP (Tabla 3).

Tabla 3: Resultados valor peróxido y acidez

\begin{tabular}{|l|c|c|l|l|}
\hline \multirow{2}{*}{ Parámetro } & \multicolumn{2}{|c|}{ Resultado } & \multirow{2}{*}{ Unidades } & \multirow{2}{*}{ Método } \\
\cline { 2 - 3 } & Aceite virgen & Aceite usado DP & & \\
\hline Valor peróxido & 1,22 & 14,97 & mEq peróxido/kg & AOAC 965.33 \\
\hline Acidez & 0,338 & 0,337 & \% Ácido oleico & \multirow{2}{*}{ ISO 660:2009 } \\
\hline
\end{tabular}


Por el contrario, el valor peróxido arrojo un aumento significativo, el aceite virgen tiene VP de 1,22 mEq peróxido $/ \mathrm{kg}$, mientras que el valor del aceite sometido a DP fue $14,97 \mathrm{mEq}$ peróxido/ $\mathrm{kg}$. Adicionalmente se realizó un ensayo calentando el aceite virgen a $75^{\circ} \mathrm{C}$ durante una hora en presencia de aire para analizar únicamente el efecto de la temperatura el valor arrojado de VP fue 4,16 mEq peróxido/kg. Se confirma que hay inicio de degradación del aceite debido a la fricción, la temperatura y la presencia de oxígeno durante el ensayo de DP, factores mencionados anteriormente los cuales contribuyen a la formación de radicales libres que reaccionan con el oxígeno para formar un radical peroxi como un producto intermedio, este radical peroxi luego ataca otra molécula lipídica para formar un hidroperóxido y otro radical libre, propagando así el proceso de oxidación (Jagadeesh y Satish, 2012).

\section{CONCLUSIONES}

Las esferas lubricadas con aceite de ajonjolí presentan el menor diámetro promedio de la huella de desgaste y el menor coeficiente de fricción; es decir, el aceite de ajonjolí tiene mayor poder lubricante que el aceite mineral 360. Adicionalmente, el aceite de ajonjolí tiene mejor desempeño en condiciones de extrema presión, presentando una carga anterior al desgaste visible y punto de soldadura mayor que el aceite mineral. La naturaleza polar de las moléculas de triglicéridos presentes en el aceite vegetal le da mayor afinidad por las superficies metálicas y en consecuencia mayor lubricidad que los aceites minerales no polares, formados por hidrocarburos alifáticos.

Con la técnica de espectroscopia IR no fue posible detectar el inicio de la degradación oxidativa de los aceites después del ensayo de desgaste preventivo, debido a que no se observa variación en los espectros de los aceites puros y los aceites usados. Se evidencia cualitativamente la presencia de metales en el aceite usado en la prueba de desgaste preventivo mediante la técnica de absorción atómica. La técnica DSC permite verificar la disminución en la estabilidad oxidativa del aceite vegetal después de la prueba de DP.

El valor peróxido del aceite de ajonjolí aumenta luego del ensayo de DP debido a la formación de radicales libres generados por las condiciones del ensayo (fricción, temperatura y presencia de aire).Esta prueba confirma el inicio de la degradación del aceite vegetal.

El aceite de ajonjolí es una materia prima de interés para la formulación de lubricantes verdes y los resultados de este trabajo son el punto de partida para estudios posteriores que buscaran mejorar las propiedades de estabilidad oxidativa y poder lubricante del aceite de ajonjolí.

\section{AGRADECIMIENTOS}

Los autores agradecen al Químico M. Sc. Nelson Núñez su colaboración durante la investigación.

\section{REFERENCIAS}

Abou-Gharbia Hany Aly, Shehata Adel, Shahidi Fereidoon, Effect of processing on oxidative stability and lipid classes of sesame oil, Food Research International, 33, 331-340 (2000).

Alves, S. M. y otros cuatro autores, Tribological behavior of vegetable oil-based lubricants with nanoparticles of oxides in boundary lubrication conditions, Tribology International, 65 (0), 28-36 (2013).

Anwar, F., Shahid, S.A. y Hussain, A.I., Assessment of oxidative deterioration of soybean oil at ambient and sunlight storage, Grasas y aceites, 58 (4), (2007).

ASTM D445-12, Standard Test Method for Kinematic Viscosity of Transparent and Opaque Liquids (and Calculation of Dynamic Viscosity), 1-5, ASTM International, (2012).

ASTM D4172-94, Standard Test Method for Wear Preventive Characteristics of Lubricating Fluid (Four-Ball Method), 1-5, ASTM International, (2010).

ASTM D2783-03, Standard Test Method for Measurement of Extreme-Pressure Properties of Lubricating Fluids (Four-Ball Method), 1-5, ASTM International, (2009).

Barrera Arellano Daniel, Estabilidad y utilización de nitrógeno en aceites y grasas, Grasas y Aceites, 49(1), 55-63 (1998).

Bart, J. C., Gucciardi, E. y Cavallaro, S., Chemical transformations of renewable lubricant feedstocks. In Biolubricants Science and technology, Woodhead Publishing Limited, Vol. 46, 249-350 (2013). 
Biresaw, G., Bantchev, G. y Cermak, S., Tribological Properties of Vegetable Oils Modified by Reaction with Butanethiol, Tribol Lett, 43 (1), 17-32 (2011).

Fernández Cedi Laura Natalia, Deterioro de grasas de freído y su influencia en la calidad de papas a la francesa, Tesis de Titulación, Dpto. Ing. Alimentos, Universidad de las Américas Puebla, México (2010).

Guala María y otros tres autores, Evaluación del poder antioxidante de fracciones de aceite esencial crudo de Schinus molle L. obtenidas por destilación al vació, Información Tecnológica, 20(2), 83-88 (2009).

Guillén, M. D. y Cabo, N., Characterization of edible oils and lard by Fourier transform infrared spectroscopy. Relationships between composition and frequency of concrete bands in the fingerprint region, J Amer Oil Chem Soc, 74 (10), 1281-1286 (1997).

Guillén, M. D. y Cabo, N., Usefulness of the Frequency Data of the Fourier Transform Infrared Spectra To Evaluate the Degree of Oxidation of Edible Oils, J. Agric. Food. Chem, 47 (2), $709-719$ (1999).

Honary, L. A. y Richter, E., Biobased Lubricants Technology. In Biobased Lubricants and Greases, John Wiley \& Sons, Ltd., 91-101 (2011).

Jayadas, N. H. y Nair, K. P., Coconut oil as base oil for industrial lubricants-evaluation and modification of thermal, oxidative and low temperature properties, Tribology International, 39 (9), 873-878 (2006).

Jagadeesh K. Mannekote y Satish V. Kailas, The effect of oxidation on the tribological performance of few vegetable oils, Journal of materials research and technology, 1(2), 91-95 (2012).

Kabuya, A. y Bozet, J. L., Comparative analysis of the lubricating power between a pure mineral oil and biodegradable oils of the same mean iso grade, Tribology Series, Elsevier, Vol.30, 25-30 (1995).

Lafont Jennifer, Páez Manuel y Torres Yudi, Análisis químico de mezclas biodiesel de aceite de cocina usado y diesel por espectroscopia infrarroja, Información Tecnológica, 22(4), 35-42 (2011).

Mannekote, J. K. y Kailas, S. V., The Effect of Oxidation on the Tribological Performance of Few Vegetable Oils, J. Mater. Res. Tecnol, 1 (2), 91-95 (2012).

Minami, I., Kikuta, S. y Okabe, H., Anti-wear and friction reducing additives composed of ortho-phenylene phosphate-amine salts for polyether type base stocks, Tribology International, 31 (6), 305-312 (1998).

Menezes, P. y otros cuatro autores, Green Lubricants: Role of Additive Size, In Green Tribology, Springer Berlin Heidelberg, 265-286 (2012).

Nunes Luana S. y otros seis autores, Multi-element determination of $\mathrm{Cu}, \mathrm{Fe}, \mathrm{Ni}$ and $\mathrm{Zn}$ content in vegetable oils samples by high-resolution continuum source atomic absorption spectrometry and microemulsion sample preparation, Food Chemistry, 127, 780-783 (2011).

Pardauil, J. J. y otros cuatro autores, Determination of the oxidative stability by DSC of vegetable oils from the Amazonian area, Bioresour, Technol., 102 (10), 5873-5877 (2011).

Sathwik, K. R., Jayadas, N. H. y Kailas, S., Natural Oil-Based Lubricants, In Green Tribology, Nosonovsky, M.; Bhushan, B., Eds. Springer, Berlin Heidelberg, 287-328 (2012).

Sharma, B. K., Perez, J. M. y Erhan, S. Z., Soybean Oil-Based Lubricants: A Search for Synergistic Antioxidants, Energy \& Fuels, 21 (4), 2408-2414 (2007).

Tan, C. P. y otros tres autores, Comparative studies of oxidative stability of edible oils by differential scanning calorimetry and oxidative stability index methods, Food Chem., 76 (3), 385-389 (2002).

$\mathrm{Wu}, \mathrm{W} .-\mathrm{H} .$, The contents of lignans in commercial sesame oils of Taiwan and their changes during heating, Food Chem., 104 (1), 341-344 (2007). 
